\title{
CODIFICATION DE LA TERMINOLOGIE JURIDIQUE DANS LES DÉFINITIONS LÉGALES
}

Ivo PETRŮ

Université de Bohême du Sud, České Budějovice

\begin{abstract}
En): This study examines the interaction between the language and law in the drafting of legal norms. More specifically, it deals with the codification of legal terms in legal definitions, exploring the processes used by a legislator in their creative work. After a brief reminder of the Linguistic-Circle-of-Prague theory concerning the subject, this study proposes a certain typology of legal definitions and focuses on the linguistic analysis of specific examples. These are taken from the New Czech Civil Code, which came into force in 2014.
\end{abstract}

Keywords (En): Codification; legal terminology; legal definition.

\section{Introduction}

La triade classique du Cercle linguistique de Prague- CLP [Pražský lingvistický kroužek]: usage, norme et codification citée dans l'intitulé du quatrième volume thématique que la revue Écho des études romanes a dédié au CLP, comprend deux termes que la science du langage emprunte au droit. Il s'agit effectivement d'un thème qui retient l'attention du juriste-linguiste, puisque l'interaction de la langue et du droit au cours de l'élaboration des normes juridiques est un sujet que nous explorons depuis un certain temps. Après avoir rédigé une étude comparative portant sur les normes juridique et linguistique (PETRŮ, 2007), nous nous intéresserons cette fois à la codification. Ciblant notamment notre étude sur la méthodologie de la codification de la terminologie juridique, nous suivrons ainsi le troisième axe proposé du volume susmentionné.

Après un rappel sommaire de la théorie pragoise relative au sujet, nous aborderons la problématique de la codification lexicale et plus concrètement, nous traiterons de la codification des termes juridiques dans les définitions légales. Ainsi introduite par la théorie générale des définitions dans la tradition bimillénaire de la logique formelle, la présente étude se concentrera sur l'activité créatrice du législateur. Ce dernier est-il conscient des issues linguistiques de sa tâche ? La démarche définitoire qu'il adopte est-elle compatible avec celle préconisée par les théories linguistiques, notamment par la science lexicographique ? Et quelles sont les caractéristiques linguistiques des différents types de définitions légales?

Pour pouvoir répondre aux diverses questions qu'engendre le sujet, nous adopterons une approche contrastive à deux niveaux distincts. En premier lieu, nous essayerons de comparer les deux méthodologies de codification, à savoir celle des linguistes et plus précisément des lexicographes, avec celle des juristes qui travaillent au nom du législateur. Deuxièmement, nous fonderons notre analyse sur des exemples de définitions légales tirées des deux Codes civils français et tchèque. Le célèbre «Code Napoléon » étant déjà largement étudié dans les travaux de nos prédécesseurs depuis sa création en 1804, nous accompagnerons plutôt nos affirmations d'exemples issus du nouveau Code civil 
tchèque [Nový občanský zákoník - NOZ] ${ }^{1}$ qui est entré en vigueur en 2014, soit exactement deux cents ans après son homologue français.

Ces efforts devraient aboutir à une réflexion théorique visant à esquisser une typologie des définitions légales et à décrire leurs marques linguistiques spécifiques. Pour ce travail, nous nous appuierons notamment sur deux sources. Sur le plan général, nous nous référerons à un recueil québécois réunissant des études relatives à la norme linguistique écrites par d'éminents chercheurs francophones : BEDARD, E. ; MAURAIS, J. (eds.), La norme linguistique, Québec, 1983. Sur le plan concret de la codification du lexique juridique, nous nous inspirerons notamment des recherches du «père fondateur» de la linguistique juridique française que fut Gérard Cornu. En effet, en tant que coauteur du Nouveau Code de procédure civile et rédacteur en chef du prestigieux Vocabulaire juridique français, il est particulièrement bien placé pour nous aider dans cette tâche, et ce d'autant plus qu'il a publié en 1981 un article précisément dédié à la problématique en question : Les définitions dans la loi

\section{La théorie générale de la codification}

Le terme de codification est un polysème dont le sens premier est juridique et qui signifie selon le Larousse 1' «action de réunir en un code des lois, des règlements », c'est-à-dire de «rassembler en un seul corps des textes législatifs ou réglementaires ». Son autre sens, pertinent pour la linguistique et qui découle d'une généralisation, s'entend, toujours selon le Larousse, comme l' «action d'ériger un système cohérent de règles», c'est-à-dire «codifier l'usage de la langue $\gg^{2}$.

\subsection{La codification en linguistique}

C'est exactement dans cette seconde acception d'aménagement de la norme linguistique conçue comme « langue standard courante » ${ }^{3}$ que les représentants du CLP, avec en tête V. Mathesius et B. Havránek, utilisaient le terme « codification». Comme l'a résumé PEŠEK (2007 : 215), ils le comprenaient en tant qu' «enregistrement de la norme contemporaine [...] sous forme de manuels

\footnotetext{
${ }^{1}$ Zákon č. 89/2012 Sb., Občanský zákoník, est le fruit de plus de dix ans de travail. Ce nouveau Code civil transforme considérablement le droit privé tchèque. Il remplace l'ancien Code civil qui datait de 1964 et qui, en dépit de ses nombreux amendements, était encore empreint de l'idéologie communiste. Long de 3081 articles, le nouveau Code civil englobe notamment le droit familial, une grande partie du code commercial et supprime la législation relative à ces domaines qui existait parallèlement.

${ }^{2} \mathrm{http}: / /$ www.larousse.fr/dictionnaires/francais/codification/16904 (consulté le 30/1/2015)

${ }^{3}$ Cette conception de la norme, qui découle très clairement des fameuses «Thèses générales du Cercle linguistique de Prague : Pour la culture de la langue », est l'un des messages les plus connus des linguistes pragois. Ledit article, qui peut être considéré comme une sorte de manifeste de l'École de Prague, est paru originellement en 1932 sous son titre tchèque Obecné zásady pro kulturu jazyka dans le recueil rédigé sous la direction de Havránek, B. et Weingart, M., Spisovná čeština a jazyková kultura. Aux fins de cette étude, nous nous baserons sur sa version française qui se trouve dans : Bédard, E.; Maurais, J. (eds.), La norme linguistique, Québec, 1983, accessible en ligne: http://www.cslf.gouv.qc.ca/bibliotheque-virtuelle/publicationhtml/?tx_iggcpplus_pi4\%5Bfile\%5D=publications/pubf101/f101app.html\#i.
} 
de grammaire, de dictionnaires et de règles d'orthoépie et d'orthographe ». La problématique fut traitée sur tous les plans linguistiques, de la prononciation à la stylistique, avec toutefois une préférence ouverte pour le domaine de l'orthographe.

Néanmoins, vu la valeur sémantique neutre du terme « enregistrement », il est important de préciser que la codification linguistique ne peut pas être comprise uniquement comme une activité objective consistant à rassembler des usages existants, mais aussi et surtout comme une activité subjective consistant à effectuer un choix entre les différentes variantes d'une langue. Se souciant avant tout de la stabilisation de la langue standard, les chercheurs regroupés au sein du CLP préconisaient des «interventions théoriques normatives » dont ils ont posé les principes généraux (Thèses CLP : 4).

Les résultats de cet interventionnisme sont, toujours selon PEŠEK, dotés d'une autorité sur le plan de la correction linguistique, car «la codification, à la différence de la norme, est explicite [...] et tout ce qui figure dans un ouvrage de codification est perçu comme correct et obligatoire » (2007 : 215). Comment expliquer le fait que la codification a donc, par rapport à la norme, une autorité d'ordre supérieur?

\subsection{La codification comme politique linguistique}

Nous pouvons observer chez certains courants sociolinguistiques, citons à titre d'exemple l'école québécoise (RONDEAU, 1983), une tendance à confondre la codification et la normalisation. Cependant, nous observons une nette différence entre ces deux processus, c'est pourquoi il nous paraît plus opportun de les distinguer. En vérité, la normalisation repose sur des normes implicites qui surgissent naturellement, car c'est «l'une des tendances spontanées des communautés linguistiques (et donc des langages), cependant que la codification consiste en un effort délibéré d'aménagement» (MOREAU, 1997: 214). La codification conduit ainsi à la production de normes explicites. En lui reconnaissant cette qualité inhérente, nous nous éloignons du domaine purement descriptif de la théorie linguistique et nous arrivons sur le champ prescriptif de la politique linguistique, qui cherche à administrer l'utilisation d'une langue.

Que les représentants du CLP ne se contentaient pas de théoriser, mais qu'ils aient œuvré pour une réelle unification de la langue tchèque est un fait bien connu. L'une des meilleures illustrations, bien qu'un peu plus tardive, en est le célèbre Dictionnnaire de la langue standard tchèque [Slovník spisovného jazyka českého]. La méthode de travail de l'équipe de lexicographes réunis autour de Havránek au sein de l'Institut pour la langue tchèque auprès de l'Académie des sciences tchécoslovaques [Ústav pro jazyk český Československé akademie věd] consistait à sélectionner certains usages considérés comme standard puisque répondant à un certain idéal présumé de la norme pour en éliminer d'autres, considérés comme impropres.

Nous pouvons donc tirer de cela la conclusion partielle qu'au sens linguistique « la codification est une activité interventionniste par laquelle un organisme doté d'une autorité administrative relève certains usages considérés comme normaux dans l'intention de les imposer comme normatifs ». Pratiquée ainsi, la codification 
doit être considérée, à l'instar de l'Académie française, comme une activité relevant de la politique linguistique.

\section{La codification du lexique}

Dans les années trente du $20^{\mathrm{e}}$ siècle, lorsque l'École de Prague a connu son essor, l'aspect lexical de la codification était délibérément mis à part dans les travaux de ses représentants, car «dans ce processus de 'codification' qui consiste essentiellement à relever ce qui existe [...] il est impossible de déterminer la signification des mots sans tenir compte de leur différenciation fonctionnelle » (Thèses CLP : 7). Par conséquent, ils préféraient, pour ce qui relève du lexique, les descriptions théoriques aux interventions normatives, à l'exception significative de la terminologie technique, car «si la terminologie technique requiert une délimitation précise des significations, cette exigence ne s'applique pas au vocabulaire standard en général » (ibidem).

\subsection{La codification lexicographique}

Cette dernière remarque, datant de 1932, reste-t-elle toujours pertinente ? La situation a considérablement évolué depuis la période précédant la Seconde Guerre mondiale. Les travaux d'Eugen Wuster ont entrainé l'institutionnalisation de l'activité terminologique, tant au niveau mondial avec l'Organisation internationale de normalisation (ISO), qu'au niveau européen avec le Comité européen de normalisation (CEN), de pair avec le développement du traitement automatique des langues, notamment dans un objectif de traduction. Par conséquent, les études terminologiques ont pris énormément d'importance et cela même au sein du vocabulaire standard.

Les continuateurs de la première génération du CLP s'en sont bien rendu compte comme en témoigne le Dictionnaire de la langue standard tchèque susmentionné et dont la première édition date de 1960 - les significations de ses plus de 192000 entrées y sont précisément délimitées. Lors de sa préparation, ses auteurs ont évidemment été confrontés aux diverses interrogations que pose la science lexicographique et que nous pourrions résumer en paraphrasant REY (1983) avec deux questions lapidaires : que définir et comment ?

De nos jours, la problématique de la codification lexicographique, bien que pouvant sembler marginale aux «pères fondateurs » du CLP, s'est hissée au premier rang des préoccupations linguistiques, en raison de l'utilité pratique d'une telle activité. Établir des définitions lexicographiques, que nous comprenons aux fins de cette étude telles que décrites par DUBOIS (2007: 131) comme «analyse sémantique du mot d'entrée [...] constituée d'une série de paraphrases synonymiques du mot d'entrée, chaque paraphrase distincte des autres constituant un sens, ou dans la terminologie lexicographique, une acception », et par conséquent connaître les significations précises des mots est devenu une nécessité dans beaucoup de milieux.

\subsection{La codification du lexique juridique}

Cette nécessité est particulièrement vraie dans le cas du droit. Son vocabulaire, à la différence d'autres terminologies considérées comme techniques, est destiné 
à tous car «nul n'est censé ignorer la loi ». Et l'utilisation exclusive de la terminologie contenue dans la loi est obligatoire. Par conséquent, la mauvaise utilisation des termes juridiques peut avoir des conséquences néfastes pour les fautifs, typiquement sous forme de sanctions de nullité des actes juridiques concernés, d'où l'utilité évidente d'une étude concernant la codification de ce vocabulaire et pas seulement pour la linguistique.

La terminologie juridique est ordinairement perçue par les linguistes comme un ensemble lexical appartenant au domaine du droit et rassemblé sous forme de définitions des entrées dans les dictionnaires et assimilés. Par exemple, le Vocabulaire juridique susmentionné comprend quelque 10000 mots (simples ou composés) ayant un ou plusieurs sens au sein du droit, c'est-à-dire des acceptions juridiques. Mais comment ces acceptions acquièrent-elles, comme nous l'avons vu plus haut, leur autorité présupposée ?

En effet, les unités lexicographiques juridiques ne peuvent acquérir cette autorité ailleurs que dans les définitions légales des termes. Ces définitions autoritaires sont porteuses des seuls sens codifiés, car prescrites par le législateur. C'est en quoi, comme le souligne CORNU (1981: 79), elles diffèrent substantiellement des définitions lexicographiques qui «ouvertes à la polysémie et davantage soumises aux usages de langue [...] cherchent à recueillir les multiples sens d'un même mot, compte tenu des usages consacrés». Les définitions légales privilégient au contraire une signification du mot parmi d'autres et cela dans l'intention de "dissiper l'équivoque et l'obscurité », continue Cornu au même endroit. En fait, la technique législative de la création des définitions juridiquement contraignantes est le moyen le plus efficace de lutte contre la polysémie, source inéluctable de confusion dans la communication ${ }^{4}$.

\section{Les définitions dans la loi}

Dans la théorie juridique, plusieurs catégories de définitions sont distinguées, chacune ayant une certaine valeur au sein du droit. La gamme va, dans l'ordre décroissant en fonction de leur validité, des définitions légales que nous traiterons en détail plus tard, aux définitions jurisprudentielles ou prétoriennes et enfin aux définitions doctrinales ou scientifiques. Il est important de souligner que ces trois types s'entrecroisent, s'influencent mutuellement. Les définitions dans la loi procurent les seules significations des termes juridiquement contraignantes et leur exacte application est sous le contrôle de la justice. Celle-ci élabore elle-même d'autres définitions dans un processus de «finition» des définitions légales, en précisant leur portée dans les cas concrets. Enfin, les créations doctrinales servent souvent au législateur, ainsi qu'au juge, comme « puits d'inspiration ».

\footnotetext{
${ }^{4}$ Nous avons présenté en détail la théorie cornuenne de polysémie dans le vocabulaire juridique dans : PETRŮ Ivo (2013), La polysémie : élément majeur de la terminologie juridique selon G. Cornu. Un exemple récent du traitement législatif de ce phénomène en droit tchèque. Écho des études romanes, České Budějovice, p. 47-57.
} 


\subsection{La typologie logique des définitions légales}

L'opération de «délimitation des significations » du CLP se transcrit depuis Aristote par la formule logique suivante : A definiendum $={ }_{\mathrm{df}} \mathrm{B}$ definiens. C'est-àdire qu'une définition instaure une équivalence entre le mot défini et sa paraphrase définissante. Ou, pour nous inscrire plutôt dans la lignée saussurienne, entre la forme du mot (signifiant) et son contenu intellectuel (signifié). Les procédés que prend cette opération logique varient, néanmoins cela se fait habituellement par référence d'abord au genre prochain (genus proximum) du mot défini, pour spécifier ensuite les traits distinctifs de son espèce (differentia specifica).

Traditionnellement, deux variantes de définitions sont distinguées dans la logique formelle. Si la référence est faite aux «propriétés essentielles de la chose désignée par le mot », la définition est dite réelle. Au contraire, lorsque le sens du mot est déterminé arbitrairement par «convention relative au sens du mot», il s'agit de la définition nominale ${ }^{5}$. Cette distinction vaut aussi pour les définitions lexicales, voire lexicographiques: le Dictionnaire de linguistique connaît la définition référentielle ou ostensive d'une part et la définition sémantique ou logique de l'autre. La première se construit «par référence à la chose que le signe dénote », tandis que la seconde "par le moyen de signes appartenant à un système construit, à une langue artificielle ou métalangue » (DUBOIS, 2007 : 131).

La même ligne de démarcation peut être tracée dans le domaine des définitions légales. En France, CORNU différencie «les définitions qui portent sur le grain des choses et celles qui portent sur la paille des mots; ou [...] entre la définition directe des choses et celle des mots. » (1981: 81). Il appelle les premières définitions réelles et les secondes définitions terminologiques. Ces deux types principaux de définitions légales n'ont pas la même portée. La définition réelle ne connaît pas de limite spatiotemporelle, car elle exprime une notion juridique à laquelle elle correspond dans l'ensemble du système juridique. Tandis que la définition terminologique indique le sens dans lequel un terme doit être pris dans l'application d'une loi concrète, comme une sorte de préinterprétation de cette loi.

La classification susmentionnée s'applique aussi en République tchèque où les ressources les plus autoritaires de la théorie juridique reprennent simplement la distinction aristotélicienne entre les définitions réelles et nominales (KNAPP, 1995) toutefois, n'oublie pas de constater avec justesse qu'en droit toute définition est en fin de compte nominale, car ce qui est décisif, même en se référant à une réalité existante, c'est la prescription de ce qu'on doit entendre devant la loi sous l'écorce du terme utilisé (1995 : 123).

\subsection{La typologie formelle des définitions légales}

Les définitions classiques dans lesquelles le definiendum est relié à son definiens, typiquement par l'intermédiaire du verbe être, abondent sur les pages des deux Codes civils. Nous pourrions les appeler « définitions stricto sensu » par opposition aux autres définitions qui ne répondent pas aux prémisses d'Aristote et que nous pourrons donc appeler «définitions largo sensu». À quoi faisons-nous

5 http://www.larousse.fr/dictionnaires/francais/d\%C3\%A9finition/22700/locution (consulté le $30 / 1 / 2015)$ 
allusion? Répondons avec CORNU (1981: 80) que «le législateur use, assez souvent, du procédé de l'énumération, limitative ${ }^{6}$ ou énonciative ${ }^{7}$. Il opère des classifications ${ }^{8}$. Il procède parfois par assimilation ${ }^{9}$. » Ces techniques législatives sortent du cadre restreint de la définition aristotélicienne, néanmoins cela n'exclut nullement leur popularité auprès des créateurs des normes juridiques.

Mais même au sein des définitions «stricto sensu », nous assistons à une diversité inattendue. Le chercheur doit s'intéresser aussi aux définitions synthétiques qui, à la différence des définitions classiques, n'analysent pas le concept en question par l'abstraction des traits caractéristiques inhérents, mais au contraire lui rattachent les traits distinctifs de dehors ${ }^{10}$. Il y a aussi les définitions génétiques qui, au lieu de définir explicitement comment on devrait entendre le terme usé par la référence à son genre et la spécification de son espèce, décrivent le mode de création d'un institut juridique ${ }^{11}$. N'oublions pas non plus les définitions contextuelles ${ }^{12}$ qui permettent de dégager le sens légal du terme en fonction du contexte et dont le cas quelque peu extrême, mais très fréquent, sont les définitions a rubrica. Ces dernières définissent implicitement, par la description des composantes de l'acte juridique en question, le terme utilisé en intitulé de la rubrique - procédé typique par exemple dans le domaine des contrats.

Cette énumération n'est pas limitative et nous pourrions établir diverses classifications des définitions légales triées selon différents points de vue. Sachant toutefois que toutes les définitions «sont au moins des instruments de clarification » (CORNU, 1981 : 98), il serait peut-être plus intéressant d'examiner leur apparence linguistique.

\section{Les définitions légales sur le plan linguistique}

Le langage juridique est fortement marqué par sa fonction prescriptive qui se reflète sur la nature linguistique des textes du droit. Les règles juridiques sont souveraines, générales et obligatoires et ces qualités se manifestent surtout dans les textes des lois créés par le législateur. Examinons dès lors les moyens linguistiques exprimant ces qualités dans les définitions légales.

\footnotetext{
${ }^{6}$ Voir $\S 18$ NOZ limitant, comme en droit français, le nombre de «osoby [personnes juridiques]» à deux : «fyzická [physique] » et «právnická [morale] ».

7 Voir \& 3 (2) NOZ énonçant quelques-uns des plus importants «zásady soukromého práva [principes du droit privé] » et invitant à compléter leur liste par l'utilisation du mot «zejména [notamment] ».

${ }^{8}$ Voir la Section 2 du Titre IV du Premier livre du NOZ sur la classification des biens ( $\$ 496-503$ ).

${ }^{9}$ Voir § $22 \mathrm{NOZ}$ qui après avoir défini «osoby blizké [personnes proches] » par énumération, en assimilent d'autres à condition qu'elles remplissent les critères précisés.

${ }^{10} \mathrm{C}$ 'est ainsi que « otec [père] » peut être retrouvé grâce aux 3 présomptions réfragables légales. Il a soit la qualité du conjoint légal, soit il se fait reconnaître comme père devant le juge ou enfin sa qualité paternelle est due à la relation sexuelle qu'il a eue avec la mère dans la période légale ( $\$ 776$ et suivants NOZ).

${ }^{11}$ Voir $§ 774 \mathrm{NOZ}$ sur «švagrovství [affinité] », relation de parenté indirecte, qui prend naissance à la suite du mariage.

${ }^{12}$ Restant dans le domaine du Droit de la famille, nous devons constater que cela concerne le mot « rodina [famille] » lui-même : utilisé dans NOZ à plusieurs endroits, la définition explicite manque.
} 


\subsection{L'énoncé des définitions légales}

Nous avons déjà mentionné que le moyen linguistique le plus utilisé pour établir l'équivalence entre le terme et sa signification est le verbe être. Le législateur en connaît pourtant d'autres et CORNU (1981: 80) cite, par exemple, les formules utilisées dans le Code civil des Français (sic) : "est censé $X$, est réputé $X$, est dénommé $X$; on appelle ainsi $X$, on entend par $X$; $X$ signifie, $X$ désigne, $X$ englobe ; $X$ constitue, $X$ forme, $X$ inclue, etc. ». Des verbes explicitant la volonté souveraine du législateur du type devoir, pouvoir, permettre ou interdire abondent également, surtout dans les « définitions largo sensu » telles que les énumérations.

L'éventail des expressions usitées du côté tchèque est riche également. Il apparaît que la variante certainement la plus fréquente dans le $\mathrm{NOZ}$ « $X$ je $\left[\mathrm{X}\right.$ est] $»^{13}$ établit des définitions réelles, tandis que la variante «X se rozumí [on entend par X] $\gg^{14}$ s'utilise plutôt dans les définitions nominales. Ces deux options principales alternent avec d'autres telles que «jedná se o $X$ [il s'agit de $\mathrm{X}] »^{15}$ ou «X tvoři [X consiste en] ${ }^{16}$ dans les définitions ordinaires ou bien avec des verbes comme «X vytváríl / zakládá [X est formé par / est constitué par] ${ }^{17}$ dans les définitions génétiques. Les constructions verbales plus complexes établissent des présomptions et des fictions juridiques. La formule «má se za to, že [est présumé que] ${ }^{18}$ introduit une présomption réfragable, tandis que la formule «platí, že [est certain que] ${ }^{19}$ introduit son opposé et la formule «považuje se za [est considéré que] ${ }^{20}$ instaure une fiction juridique.

Dans ce contexte, il n'est pas sans intérêt non plus de signaler que nous pouvons observer dans les deux codes civils les formes négatives de pratiquement toutes ces variantes des énoncés des définitions légales ${ }^{21}$. En effet, les définitions dans la loi ne prennent pas seulement la forme affirmative, mais très souvent la forme de négation ce qui est normalement exclu dans la logique formelle.

\subsection{L'analyse linguistique de ces énoncés}

Nous pouvons remarquer que tous les exemples des verbes typiques des définitions légales françaises ou tchèques s'utilisent d'habitude à la troisième personne du singulier et au présent de l'indicatif. La classification du mode verbal est pourtant trompeuse, la doctrine préfère le désigner comme l'indicatif déontique, puisqu'il a valeur d'impératif. En fait, dans les deux cas, il s'agit des marques typiques de neutralité du législateur souverain qui forgent des règles générales applicables obligatoirement à tous.

\footnotetext{
${ }^{13}$ Voir $\$ 775$ NOZ sur «matka [mère] » qui, à la différence du père, est toujours certaine, car définie par référence au genre prochain et par spécification de l'espèce : c'est la femme qui a mis l'enfant au monde.

${ }^{14}$ Voir $\$ 606$ NOZ sur «polovina mésíce [moitié d'un mois] ».

${ }^{15}$ Voir $\$ 503$ (2) NOZ sur « odštěpný závod [succursale]».

${ }^{16}$ Voir $\$ 495$ NOZ sur «majetek [patrimoine] ».

17 Voir $\S 210 \mathrm{NOZ}$ sur la la formation d'une «korporace [corporation]» et $\S 306 \mathrm{NOZ}$ sur la constitution d'une «nadace [fondation] ».

18 Voir les paragraphes précités définissant le père.

${ }^{19}$ Voir $§ 115$ sur «pitva [autopsie] ».

${ }^{20}$ Voir $\$ 700$ sur « rodinný závod [entreprise familiale] ».

${ }^{21}$ Voir $\$ 494$ explicitant que «zvíre [animal] »n'est pas un bien.
} 
De l'autre côté, la valeur générale de la règle est encore accentuée par des procédés comme l'emploi régulier de l'article défini marquant la portée universelle de la définition et du genre masculin. Le sujet masculin du definiendum tel que «le propriétaire » désigne évidemment toute personne sans distinction de sexe. Sans parler de l'utilisation en français comme en tchèque des termes indéfinis, pronoms et adjectifs affirmatifs comme «vše [tout] » ${ }^{22}$ ou «každý [chacun] $»^{23}$, négatifs comme «nikdo [nul] $»^{24}$ ou «žádný [aucun]» ${ }^{25}$, ainsi qu'indéterminés comme «kdo/kdokoli [quiconque] ${ }^{26}$ ou «jiná osoba [autrui] ${ }^{27}$.

Sur le plan syntaxique, nous pouvons observer dans les exemples présentés cidessus la surutilisation des constructions impersonnelles et de la voix passive. Dans les définitions légales françaises s'y ajoute une autre particularité linguistique par rapport à la langue standard qui est le recours fréquent à l'inversion syntaxique comme en témoignent les formules susmentionnées du type « est réputé $\mathrm{X}$ ».

\subsection{L'approche du législateur}

Nous avons pu constater précédemment que le législateur a certaines préférences dans sa façon de manier la langue. En dépit de celles-ci, nous pourrions admettre à première vue qu'il ne s'écarte pas excessivement au regard des habitudes linguistiques normales. Toutefois, à y regarder de plus près l'aspect sémantique des termes juridiques, il faut nuancer la première impression. L'approche du législateur « consiste à forger un langage codé pour la lecture d'un texte» (CORNU, 1981:87), en l'occurrence un texte de la loi. Par conséquent le législateur, en forgeant le sens légal de la terminologie usitée, s'éloigne parfois, notamment lors de l'élaboration des définitions dites nominales ou terminologiques, des entendements ordinaires de certains mots. "Le législateur peut restreindre ou étendre le sens du mot, relativement à sa signification ordinaire; spécifier ou généraliser [...] 》 (ibid). Grâce aux définitions légales, ces mots deviennent ainsi de vrais termes juridiques avec des significations délimitées et obligatoires pour les besoins du droit.

Il faut donc comprendre la définition légale comme une "opération consistant à fixer le sens que l'on veut donner à un mot» (ibidem). En fixant ce sens arbitraire, le législateur jouit d'une liberté incomparable par rapport à son homologue lexicographe. Ce dernier, en cherchant des sens possibles des mots, ne peut puiser que dans ce qui est normal : il suit la norme ; tandis que le législateur décide ce qui sera normatif : il établit la norme. En fait, comme le souligne CORNU

\footnotetext{
${ }^{22}$ Voir $§ 489 \mathrm{NOZ}$ sur «věc [bien] ».

${ }^{23}$ Voir § 6 (1) NOZ établissant «povinnost každého poctivě jednat [obligation de chacun d'agir honnêtement] ».

${ }^{24}$ Voir § 6 (2) NOZ établissant le principe complémentaire «nemo turpitudinem suam allegare potest $=$ nikdo nesmí těžit ze svého nepoctivého činu $[$ nul ne peut se prévaloir de sa propre turpitude] ».

${ }^{25}$ Voir $\$ 1634 \mathrm{NOZ}$ sur « odúmrt' [déshérence] ».

${ }^{26}$ Voir $\$ 420 \mathrm{NOZ}$ sur «podnikatel [entrepreneur] ».

${ }^{27}$ Voir § 1012 NOZ sur les prérogatives du propriétaire comprenant entre autres le droit d'éliminer « jiné osoby [autrui] » de la disposition avec l'objet de propriété.
} 
(ibidem) «toute définition légale est une règle de droit, elle constitue une norme juridique, un énoncé de droit positif».

Les définitions légales sont dès lors très puissantes. Ayant toujours ce caractère de norme juridique, elles doivent être respectées sous la menace d'application des sanctions. Mais leur puissance sur le plan linguistique repose ailleurs. Leur mérite indubitable est de «livrer le sens d'un terme, de placer un signifié sous chaque signifiant, de déterminer la compréhension d'un terme, le contenu du contenant » (CORNU, $1981: 85)$

\section{Conclusion}

Force est de constater qu'il existe deux approches de codification dont la première est juridique et la seconde est linguistique. Dans les deux cas, il s'agit d'une activité intentionnelle de création des normes explicites dont l'objectif commun est la stabilisation des phénomènes sociaux en question qui sont le droit d'une part et la langue de l'autre.

Reconnaissant cette similitude globale, après avoir étudié les deux approches codificatrices sur le plan de la terminologie juridique, nous pouvons constater la différence fondamentale quant à la méthodologie des deux approches. Le législateur agit en maître en son domaine et son approche est libre, puisque dépourvue de contraintes objectives - son discours est politique. Alors que le lexicographe agit en observateur neutre et son approche est défensive, puisque soumise aux limites posées par le fonctionnement naturel d'une langue - son discours se veut scientifique. Par conséquent, la méthode privilégiée du premier est la prescription de la norme, il détermine l'unique signification des notions juridiques dans les définitions légales. Alors que la stratégie favorite du second est la description des usages, il recueille tous les sens potentiels des mots dans les dictionnaires.

Les fruits de la codification juridique, telle que nous l'avons étudiée dans cet article, se manifestent sous la forme des définitions contenues dans nos lois. Ces définitions représentent la matière authentique offerte au lexicographe pour la recherche consécutive, et cette recherche ne devrait pas avoir pour ambition de créer la norme, mais de l'étudier dans un premier temps pour pouvoir l'enseigner par la suite. Car toute production scientifique, y compris les dictionnaires, est en fin de compte l'instrument de la connaissance.

\section{BIBLIOGRAPHIE}

CORNU Gérard (1981), Les définitions dans la loi, Mélanges dédiés à Jean Vincent, Paris, Dalloz, p. 77-92.

CORNU Gérard (2005), Linguistique juridique, Paris, Montchrestien.

CORNU Gérard et al. (2004), Vocabulaire juridique, Paris, PUF.

DUBOIS Jeanet al. (2007), Linguistique \& sciences du langage, Paris, Larousse.

HAVRÁNEK Bohuslav et al. (1989), Slovník spisovného jazyka českého, $2^{\mathrm{e}}$ éd.

Praha, Academia. Accessible en ligne : http://ssjc.ujc.cas.cz/ 
KNAPP Viktor (1995), Teorie práva, Praha, C.H.Beck.

KNAPP Viktor; GERLOCH Aleš (2001), Logika v právním myšlení, Praha, Eurolex Bohemia.

Larousse, dictionnaire de français, en ligne: www.larousse.fr

MorEAU Marie-Louise et al. (1997) Sociolinguistique : concepts de base, Liège, Mardaga.

PEŠEK Ondřej (2007), Dynamique, norme et codification - qu'en est-il aujourd'hui de la théorie pragoise? Norme, normativité, transgression, Lask, Oficyna Wydawnicza LEKSEM, p. 213-220.

PETRŮ Ivo (2007), Comparaison de la norme juridique avec la norme linguistique, Norme, normativité, transgression, Lask, Oficyna Wydawnicza LEKSEM, p. 221-226.

PETRŮ Ivo (2013), La polysémie : élément majeur de la terminologie juridique selon G. Cornu. Un exemple récent du traitement législatif de ce phénomène en droit tchèque. Écho des études romanes, České Budějovice, p. 47-57.

REY Alain, Norme et dictionnaires, in: Bédard, E. ; Maurais, J. (eds.), La norme linguistique, Gouvernement du Québec, 1983, accessible en ligne: http://www.cslf.gouv.qc.ca/bibliotheque-virtuelle/publication$\mathrm{html} /$ ?tx_iggcpplus_pi4\%5Bfile\%5D=publications/pubf101/f101p4a.html\#xvi

RONDEAU Guy (1983), La normalisation linguistique, terminologique et technique au Québec, in: Bédard, E.; Maurais, J. (éds.), La norme linguistique, Gouvernement du Québec, accessible en ligne: http://www.cslf.gouv.qc.ca/bibliotheque-virtuelle/publicationhtml/?tx_iggcpplus_pi4\%5Bfile\%5D=publications/pubf101/f101p4a.html\#xvi

Thèses générales du Cercle linguistique de Prague: Pour la culture de la langue [Obecné zásady pro kulturu jazyka], in : Bédard, E. ; Maurais, J. (éds., 1983), La norme linguistique, Gouvernement du Québec, accessible en ligne : http://www.cslf.gouv.qc.ca/bibliotheque-virtuelle/publication$\mathrm{html} /$ ?tx_iggcpplus_pi4\%5Bfile\%5D=publications/pubf101/f101 app.html\#i

Zákon č. 89/2012 Sb., Občanský zákoník, accessible en ligne : http://www.obcanskyzakonik.justice.cz 